\title{
Monopole operators and mirror symmetry in three dimensions
}

\author{
Vadim Borokhov, Anton Kapustin and Xinkai Wu \\ California Institute of Technology \\ Pasadena, CA 91125, USA \\ E-mail: borokhov@theory.caltech.edu, kapustin@theory.caltech.edu, \\ xinkaiwu@theory.caltech.edu
}

ABSTRACT: We study vortex-creating, or monopole, operators in 3d CFTs which are the infrared limit of $N=2$ and $N=4$ supersymmetric QEDs in three dimensions. Using large- $N_{f}$ expansion, we construct monopole operators which are primaries of short representations of the superconformal algebra. Mirror symmetry in three dimensions makes a number of predictions about such operators, and our results confirm these predictions. Furthermore, we argue that some of our large- $N_{f}$ results are exact. This implies, in particular, that certain monopole operators in $N=4 d=3$ SQED with $N_{f}=1$ are free fields. This amounts to a proof of $3 \mathrm{~d}$ mirror symmetry in a special case.

Keywords: Field Theories in Lower Dimensions, 1/N Expansion, Duality in Gauge Field Theories, Supersymmetry and Duality, 


\section{Contents}

1. Introduction 1

2. Monopole operators in $N=2 d=3$ SQED 3

2.1 Review of $N=2 \mathrm{SQED}$ and $N=2$ mirror symmetry

2.2 Monopole operators in $N=2$ SQED at large $N_{f}$

2.3 A comparison with the predictions of $N=2$ mirror symmetry 10

3. Monopole operators in $N=4 d=3$ SQED

3.1 Review of $N=4$ SQED and $N=4$ mirror symmetry

3.2 Monopole operators in $N=4 \mathrm{SQED}$ at large $N_{f}$

3.3 A comparison with the predictions of $N=4$ mirror symmetry

4. Beyond the large- $N_{f}$ limit 17

4.1 Non-renormalization theorems for the anomalous charges 17

4.2 A derivation of the basic $N=4$ mirror symmetry 18

5. Discussion of results and open problems 18

A. Radial quantization of $N=2$ SQED 19

\section{Introduction}

One of the most remarkable exact results in quantum field theory is the equivalence of the quantum sine-Gordon model and the massive Thirring model [1, 2]. The "duality" between these two theories has a very transparent physical meaning. Quantum sine-Gordon theory contains topological solitons (kinks). It turns out that a certain operator which has nonzero matrix elements between the vacuum and the one-kink sector is a fermion and satisfies the equations of motion of the massive Thirring model [2]. Thus the duality arises from "rewriting" the sine-Gordon model in terms of kink variables.

In the last two decades a large number of dualities have been proposed for quantum field theories in higher dimensions. The first successful proposal of this sort is the S-

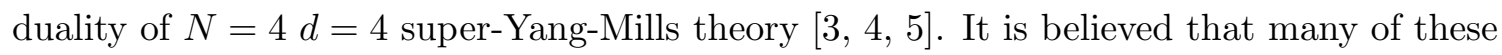
conjectural dualities have the same origin as the sine-Gordon/Thirring duality, i.e. they arise from "rewriting" a theory in terms of new fields which create topological solitons. But so far nobody managed to prove a non-trivial higher-dimensional duality along the lines of [2]. The main reason for this is that the conjectured dualities in higher dimensions 
typically involve non-abelian gauge theories and are vastly more complicated than the sineGordon/Thirring duality. Usually, it is not even clear which solitons are "responsible" for the duality.

In this paper we report a progress in proving a non-perturbative duality in three dimensions. This duality, known as $3 \mathrm{~d}$ mirror symmetry, has been proposed by K. Intriligator and N. Seiberg [6], and later studied by a number of authors [7-[21]. Mirror symmetry in three dimensions has a number of special features that make it more amenable to study than other higher-dimensional dualities. First of all, mirror symmetry makes sense for abelian gauge theories, for which the complications due to the presence of unphysical degrees of freedom are not so severe. Second, it is known how to construct a mirror theory (in fact, many mirror theories [16]) for any abelian gauge theory [10, 16. The mirror is always an abelian gauge theory, but usually with a different gauge group. Third, all mirror pairs can be derived from a certain "basic" mirror pair by formal manipulations [16. This basic example identifies the infrared limit of $N_{f}=1 N=4 d=3$ SQED with a free theory of a twisted hypermultiplet. To prove this basic example of mirror symmetry, one only needs to construct a twisted hypermultiplet field out of the fields of $N=4$ SQED and show that it is free. Fourth, it is known what the relevant topological soliton is in this case: it is none other than the Abrikosov-Nielsen-Olesen vortex [12].

In our previous paper [22], we showed how to define vortex-creating (or monopole) operators in the infrared limit of $3 \mathrm{~d}$ abelian gauge theories. The main tools used were radial quantization and large- $N_{f}$ expansion. The only example considered in [22] was ordinary (non-supersymmetric) QED. In this theory monopole operators have irrational dimensions at large $N_{f}$ and do not satisfy any nice equation of motion. In this paper we study monopole operators in $N=2$ and $N=4$ SQEDs. More precisely, we construct monopole operators in $3 \mathrm{~d}$ SCFTs which are the infrared limit of $N=2$ and $N=4$ SQEDs. We focus on operators which live in short multiplets of the superconformal algebra. The dimensions of primaries of such multiplets saturate a BPS-like bound, so we will sometimes refer to operators in short multiplets as BPS operators.

Mirror symmetry makes predictions about the spectrum and other properties of BPS operators, including those with non-zero vortex charge. In [12 some of these predictions have been verified on the Coulomb branch of $N=2 \mathrm{SQED}$, where the infrared theory is free. Our computations are performed at the origin of the moduli space, where the infrared theory is an interacting SCFT. Thus the agreement between our results and the predictions of mirror symmetry is a new check of this duality. In addition, we have been able to verify certain interesting relations in the chiral ring which follow from mirror symmetry. In the approach of [12], the origin of these relations was obscure.

In many cases one can go further and argue that certain results derived at large $N_{f}$ remain valid even for $N_{f}$ of order one. For example, our monopole operators have "anomalous" transformation laws under global symmetries, whose form is fixed by quasi-topological considerations (the Atiyah-Patodi-Singer index theorem). This implies that the global charges of monopole operators do not receive corrections at any order in $1 / N_{f}$ expansion. Furthermore, since our monopole operators belong to short representations of the superconformal algebra, their scaling dimensions are determined by their transformation law 
under R-symmetry. In the case of $N=4 \mathrm{SQED}$, where it is easy to identify the relevant Rsymmetry, this allows us to determine the exact scaling dimensions of monopole operators for all $N_{f}$. Our main assumption is that the $1 / N_{f}$ expansion has a large enough domain of convergence.

If we consider the special case of $N=4 \mathrm{SQED}$ with $N_{f}=1$, then the above arguments tell us that a certain monopole operator is a (twisted) hypermultiplet whose lowest component is a scalar of dimension $1 / 2$. In a unitary theory, this is only possible if the hypermultiplet is free. Thus we are able to show that for $N_{f}=1$ certain monopole operators satisfy free equations of motion. This is essentially the statement of mirror symmetry in this particular case.

The paper is organized as follows. In section 2 we study monopole operators in the infrared limit of $N=2 d=3 \mathrm{SQED}$ at large $N_{f}$ and compare with the predictions of mirror symmetry. In section 3 we do the same for $N=4 d=3$ SQED. In section $⿴$ t we show that certain large- $N_{f}$ results are exact, and argue that this implies the "basic" example of $N=4$ mirror symmetry. In section 目 we discuss our results and list open problems.

\section{Monopole operators in $N=2 d=3$ SQED}

\subsection{Review of $N=2$ SQED and $N=2$ mirror symmetry}

$N=2 d=3$ SQED can be obtained by the dimensional reduction of $N=1 d=4$ SQED. The supersymmetry algebra contains a complex spinor supercharge $Q_{\alpha}$ and its complexconjugate $\bar{Q}_{\alpha}$. The field content is the following: a vector multiplet with gauge group U(1), $N_{f}$ chiral multiplets of charge 1 and $N_{f}$ chiral multiplets of charge -1 . We will use $N=2$ superspace to describe these fields. General superfields are functions of $x \in \mathbb{R}^{2,1}$, a complex spinor $\theta_{\alpha}$, and its complex-conjugate $\bar{\theta}_{\alpha}$. The vector multiplet is described by a real superfield $V(x, \theta, \bar{\theta})$ satisfying $V^{\dagger}=V$. The corresponding field-strength multiplet is $\Sigma=\epsilon^{\alpha \beta} D_{\alpha} \bar{D}_{\beta} V$. The lowest component of $\Sigma$ is a real scalar $\chi$, while its top component is the gauge field-strength $F_{\mu \nu}$. The vector multiplet also contains a complex spinor $\lambda_{\alpha}$ (photino). A chiral multiplet is described by a superfield $Q(x, \theta, \bar{\theta})$ satisfying the chirality constraint:

$$
\bar{D}_{\alpha} Q=0 .
$$

It contains a complex scalar $A$, a complex spinor $\psi_{\alpha}$, and a complex auxiliary field $F$. We will denote the superfields describing charge 1 matter multiplets by $Q_{j}, j=1, \ldots, N_{f}$, and the superfields describing charge -1 matter multiplets by $\tilde{Q}_{j}, j=1, \ldots, N_{f}$. Then the action takes the form

$$
S_{N=2}=\int d^{3} x d^{4} \theta\left\{\frac{1}{e^{2}} \Sigma^{\dagger} \Sigma+\sum_{j=1}^{N_{f}}\left(Q_{j}^{\dagger} e^{2 V} Q_{j}+\tilde{Q}_{j}^{\dagger} e^{-2 V} \tilde{Q}_{j}\right)\right\} .
$$

Besides being supersymmetric, this action has a global $\mathrm{SU}\left(N_{f}\right) \times \mathrm{SU}\left(N_{f}\right) \times \mathrm{U}(1)_{B} \times \mathrm{U}(1)_{N}$ symmetry. The action of $\mathrm{SU}\left(N_{f}\right) \times \mathrm{SU}\left(N_{f}\right)$ is obvious (it is a remnant of the chiral flavor symmetry of $N=1 d=4 \mathrm{SQED})$. Under $\mathrm{U}(1)_{B}$ the fields $Q_{j}$ and $\tilde{Q}_{j}$ have charges 1 , 
while $V$ transforms trivially. Finally, there is an R-symmetry $\mathrm{U}(1)_{N}$ under which the fields transform as follows:

$$
\begin{aligned}
Q_{j}(x, \theta, \bar{\theta}) & \mapsto Q_{j}\left(x, e^{i \alpha} \theta, e^{-i \alpha} \bar{\theta}\right), \\
\tilde{Q}_{j}(x, \theta, \bar{\theta}) & \mapsto \tilde{Q}_{j}\left(x, e^{i \alpha} \theta, e^{-i \alpha} \bar{\theta}\right), \\
V(x, \theta, \bar{\theta}) & \mapsto V\left(x, e^{i \alpha} \theta, e^{-i \alpha} \bar{\theta}\right) .
\end{aligned}
$$

There is one other conserved current:

$$
J^{\mu}=\frac{1}{4 \pi} \epsilon^{\mu \nu \rho} F_{\nu \rho} .
$$

Its conservation equivalent to the Bianchi identity. We will call the corresponding charge the vortex charge, and the corresponding symmetry $\mathrm{U}(1)_{J}$ symmetry. All the fundamental fields have zero vortex charge; our task in this paper will be to construct operators with non-zero vortex charge and compute their quantum numbers. Operators with non-zero vortex charge will be called monopole operators.

One can add an $N=2$ Chern-Simons term to the action of $N=2$ SQED. However, the theory is consistent without it, and in this paper we will limit ourselves to the case of vanishing Chern-Simons coupling.

$N=2 d=3$ SQED is super-renormalizable and becomes free in the ultraviolet limit. In the infrared it flows to an interacting superconformal field theory (SCFT). Note that the action needs no counter-terms, if one uses a regularization preserving all the symmetries. Thus the infrared limit is equivalent to the limit $e \rightarrow \infty$.

In general, the infrared CFT is strongly coupled and quite hard to study. A simplification arises in the large $N_{f}$ limit, where the infrared theory becomes approximately gaussian. The reason for this is the same as in the non-supersymmetric case 22. At leading order in the large $N_{f}$ expansion, the matter fields retain their UV dimensions. The dimension of the gauge field strength multiplet $\Sigma$ is 1 to all orders in $1 / N_{f}$ expansion. This can be traced to the fact that the dual of the gauge field strength is an identically conserved current, as well as a primary field in the infrared SCFT. ${ }^{1}$ A well-known theorem states that in a unitary CFT in $d$ dimensions a conserved primary current has dimension $d-1$. Since the gauge field strength occurs as the top component of $\Sigma$, and $\theta, \bar{\theta}$ have dimension $-1 / 2$, this implies that the photino has infrared dimension $3 / 2$, while the lowest component $\chi$ has dimension 1 .

The IR dimensions of $Q$ and $\tilde{Q}$ can be computed order by order in $1 / N_{f}$ expansion, but the exact answer for all $N_{f}$ is unknown. The only other thing we know about these dimensions is that they are equal to the R-charges of $Q$ and $\tilde{Q}$. This is a consequence of the fact that $Q$ and $\tilde{Q}$ live in short representation of the superconformal algebra, and therefore their scaling dimensions are constrained by unitarity. ${ }^{2}$ However, the R-current

\footnotetext{
${ }^{1}$ In the UV the dual of the field strength is not a primary, but a descendant of a scalar known as the dual photon.

${ }^{2}$ Strictly speaking, it is the dimension of gauge-invariant chiral primaries like $Q \tilde{Q}$ that is constrained by unitarity to be equal to the R-charge. However, since $Q$ and $\tilde{Q}$ are chiral superfields, the dimension and R-charge of $Q \tilde{Q}$ is twice the dimension and R-charge of $Q$ and $\tilde{Q}$, and the claimed result follows.
} 
in question is not necessarily the one discussed above. Rather, it is some unknown linear combination of the $\mathrm{U}(1)_{N}$ and $\mathrm{U}(1)_{B}$ currents. We will call it the "infrared" R-current, to avoid confusion with $\mathrm{U}(1)_{N}$ current defined above. In the large $N_{f}$ limit it is easy to see that the infrared R-charge is

$$
R_{\mathrm{IR}}=N+B\left(\frac{1}{2}+O\left(\frac{1}{N_{f}}\right)\right)
$$

where $N$ and $B$ are the charges corresponding to $\mathrm{U}(1)_{N}$ and $\mathrm{U}(1)_{B}$. For $N_{f}$ of order 1 we do not know the coefficient in front of $B$, and so cannot easily determine the infrared dimensions of $Q$ and $\tilde{Q}$.

For $N_{f}=1$ mirror symmetry comes to our rescue. The statement of $3 \mathrm{~d}$ mirror symmetry in this case is that the IR limit of $N=2$ SQED is the same as the IR limit of another $N=2$ gauge theory. This other gauge theory has gauge group $\mathrm{U}(1)^{N_{f}} / \mathrm{U}(1)_{\text {diag }}$, and $3 N_{f}$ chiral matter multiplets $q_{j}, \tilde{q}_{j}, S_{j}, j=1, \ldots, N_{f}$. The action of the mirror theory has the form

$$
\begin{aligned}
S_{\text {dual }}=\int & d^{3} x d^{4} \theta \sum_{j=1}^{N_{f}}\left\{\frac{1}{e^{2}} \Sigma_{j}^{\dagger} \Sigma_{j}+\frac{1}{e^{2}} S_{j}^{\dagger} S_{j}+q_{j}^{\dagger} e^{2 V_{j}-2 V_{j-1}} q_{j}+\tilde{q}_{j}^{\dagger} e^{-2 V_{j}+2 V_{j-1}} \tilde{q}_{j}\right\}+ \\
& +\left(\int d^{3} x d^{2} \theta \sum_{j=1}^{N_{f}} q_{j} \tilde{q}_{j} S_{j}+\text { h.c. }\right)
\end{aligned}
$$

where the gauge multiplets satisfy the constraints

$$
V_{0}=V_{N_{f}}, \quad \sum_{j=1}^{N_{f}} V_{j}=0 .
$$

Note that the chiral fields $S_{j}$ are neutral with respect to the gauge group and couple to the rest of the theory only through a superpotential.

The mirror theory also flows to a strongly coupled SCFT in the infrared limit $e \rightarrow \infty$, and in general the mirror description does not help to compute the IR scaling dimensions in the original theory. However, the case $N_{f}=1$ is very special: the mirror gauge group becomes trivial, and the mirror theory reduces to the Wess-Zumino model in three dimensions with the action

$$
S_{\mathrm{WZ}}=\int d^{3} x d^{4} \theta\left(q^{\dagger} q+\tilde{q}^{\dagger} \tilde{q}+S^{\dagger} S\right)+\left(\int d^{3} x d^{2} \theta q \tilde{q} S+\text { h.c. }\right) .
$$

This theory has "accidental" $S_{3}$ symmetry permuting $q, \tilde{q}$, and $S$, which allows one to determine their infrared R-charges. Indeed, since in the infrared limit the superpotential term must have R-charge 2, the R-charges of $q, \tilde{q}$ and $S$ must be $2 / 3$. The mirror map identifies $S$ with the operator $Q \tilde{Q}$ in the original theory [12]. Thus we infer that for $N_{f}=1$ $Q$ and $\tilde{Q}$ have infrared R-charge $1 / 3$. Comparing with large- $N_{f}$ results, we see that the infrared R-charge has a non-trivial dependence on $N_{f}$.

Let us describe in more detail the matching of global symmetries between the original and mirror theories following [12]. The symmetry $\mathrm{U}(1)_{B}$ of the original theory is mapped to the symmetry under which all $S_{j}$ have charge 2 , while $q_{j}$ and $\tilde{q}_{j}$ have charges -1 . The 
symmetry $\mathrm{U}(1)_{J}$ is mapped to the $\mathrm{U}(1)$ symmetry under which all $q_{j}$ have charge $1 / N_{f}$, all $\tilde{q}_{j}$ have charge $-1 / N_{f}$, while $S_{j}$ are uncharged. The R-symmetry $\mathrm{U}(1)_{N}$ maps to an R-symmetry under which all $q_{j}$ and $\tilde{q}_{j}$ have charge 1 and $S_{j}$ are uncharged. The mapping of non-abelian symmetries is not well understood. It is only known that that the currents corresponding to the Cartan subalgebra of the diagonal $\mathrm{SU}\left(N_{f}\right)$ are mapped to the $N_{f}-1$ $\mathrm{U}(1)_{J}$ currents of the mirror theory.

\subsection{Monopole operators in $N=2$ SQED at large $N_{f}$}

Our strategy for studying monopole operators will be the same as in [22. In any $3 \mathrm{~d}$ conformal field theory, there is a one-to-one map between local operators on $\mathbb{R}^{3}$ and normalizable states of the same theory on $\mathbb{S}^{2} \times \mathbb{R}$. Therefore we will look for states with non-zero vortex charge on $\mathbb{S}^{2} \times \mathbb{R}$. In other words, we will be studying $N=2$ SQED on $\mathbb{S}^{2} \times \mathbb{R}$ in the presence of a magnetic flux on $\mathbb{S}^{2}$. Since our goal is to check the predictions of mirror symmetry, we will require that the states be annihilated by half of the supercharges; then the corresponding local operators will live in short representations of the superconformal algebra. The low-energy limit of $N=2$ SQED is an interacting SCFT, so in order to make computations possible, we will take $N_{f}$ to be very large. This has the effect of making the CFT weakly coupled. In particular, in the large $N_{f}$ limit the fluctuations of the gauge field and its superpartners are suppressed, and one can treat them as a classical background. In other words, at leading order in $1 / N_{f}$ we end up with free chiral superfields coupled to an appropriate background vector superfield. We will discuss how one can go beyond the

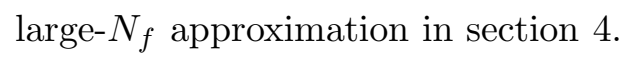

The states on $\mathbb{S}^{2} \times \mathbb{R}$ of interest to us are in some sense BPS-saturated, since they are annihilated by half of the supercharges. But in contrast to the situation in flat space, here the supercharges do not commute with the hamiltonian $\mathrm{H}$ which generates translations on $\mathbb{R}$. Indeed, since the hamiltonian on $\mathbb{S}^{2} \times \mathbb{R}$ is the same as the dilatation generator on $\mathbb{R}^{3}$, and supercharges have dimension $1 / 2$, it follows that the supercharges obey

$$
\left[\mathrm{Q}_{\alpha}, \mathrm{H}\right]=-\frac{1}{2} \mathrm{Q}_{\alpha}, \quad\left[\overline{\mathrm{Q}}_{\alpha}, \mathrm{H}\right]=-\frac{1}{2} \overline{\mathrm{Q}}_{\alpha}
$$

Note also that in the radial quantization approach $\mathrm{Q}_{\alpha}$ and $\overline{\mathrm{Q}}_{\alpha}$ are no longer hermitean conjugate of each other. Rather, their hermitean conjugates are superconformal boosts $S_{\alpha}$ and $\overline{\mathrm{S}}_{\alpha}$, which have dimension $-1 / 2$.

For the same reasons as in [22], in the large $N_{f}$ limit the energy $E$ of the states with non-zero vortex charge is of order $N_{f}$. By unitarity, for scalar states $E$ is bounded from below by the R-charge $R_{\mathrm{IR}}$. Furthermore, we will see below that in the limit $N_{f} \rightarrow \infty$ $R_{\mathrm{IR}}$ is also of order $N_{f}$, while the combination $E-R_{\mathrm{IR}}$ stays finite for all the states we encounter. A similar limit in $d=4$ SCFTs recently gained some prominence in connection with AdS/CFT correspondence [23]. But unlike [23], we take the number of flavors, rather than the number of colors, to infinity.

First let us determine which classical background on $\mathbb{S}^{2} \times \mathbb{R}$ we need to consider. As in [22], we have a gauge field on $\mathbb{S}^{2} \times \mathbb{R}$ with a magnetic flux $n$. Assuming rotational invariance of the large- $N_{f}$ saddle point, this implies that we have a constant magnetic field 
on $\mathbb{S}^{2}$. The only other bosonic field in the $N=2$ vector multiplet is the real scalar $\chi$. It is determined by the condition of the vanishing of the photino variation under half of the SUSY transformations. This will ensure that the monopole operator we are constructing is a chiral primary.

It is convenient to work out the photino variations on $\mathbb{R}^{3}$, and then make a conformal transformation to $\mathbb{S}^{2} \times \mathbb{R}$. Photino variations in euclidean $N=2$ SQED on $\mathbb{R}^{3}$ have the form

$$
\begin{aligned}
& \delta \lambda=i\left(-\sigma^{i} \partial_{i} \chi-\frac{1}{2} \epsilon^{i j k} \sigma^{k} F_{i j}+D\right) \xi \\
& \delta \bar{\lambda}=i\left(-\sigma^{i} \partial_{i} \chi+\frac{1}{2} \epsilon^{i j k} \sigma^{k} F_{i j}-D\right) \bar{\xi}
\end{aligned}
$$

where $\xi$ and $\bar{\xi}$ are complex spinors which parametrize SUSY variations. (In euclidean signature, they are not related by complex conjugation.) Since we are setting the background values of the matter fields to zero, the D-term can be dropped. Half-BPS states are annihilated by $\bar{\xi}_{\alpha} \overline{\mathrm{Q}}^{\alpha}$ for any $\bar{\xi}$ and therefore must satisfy

$$
F=-* d \chi
$$

Hence the scalar background on $\mathbb{R}^{3}$ is

$$
\chi=\frac{n}{2 r},
$$

where $n$ is the vortex charge (the magnetic charge of the Dirac monopole on $\mathbb{R}^{3}$ ). Unsurprisingly, supersymmetry requires the bosonic field configuration to be an abelian BPS monopole. Recalling that $\chi$ has dimension 1 in the infrared, we infer that on $\mathbb{S}^{2}$ the scalar background is simply a constant:

$$
\chi=\frac{n}{2} .
$$

Similarly, an anti-BPS state is annihilated by $\xi_{\alpha} \mathrm{Q}^{\alpha}$ for any $\xi$, and therefore the scalar field on $\mathbb{S}^{2}$ is

$$
\chi=-\frac{n}{2} \text {. }
$$

Having fixed the classical background, we proceed to compute the spectrum of matter field fluctuations. The details of the computation are explained in the appendix. The results are as follows. The energy spectrum of charged scalars is the same for $A^{j}$ and $\tilde{A}^{j}$, does not depend on whether one is dealing with a BPS or an anti-BPS configuration, and is given by

$$
E= \pm E_{p}= \pm\left(\frac{|n|-1}{2}+p\right), \quad p=1,2, \ldots
$$

The degeneracy of the $p^{\text {th }}$ eigenvalue is $2\left|E_{p}\right|$, and the corresponding eigenfunctions transform as an irreducible representation of the rotation group $\mathrm{SU}(2)_{\text {rot. }}$. The spectrum is symmetric with respect to $E \rightarrow-E$. 
The energy spectrum of charged spinors is the same for $\psi^{j}$ and $\tilde{\psi}^{j}$ and is given by

$$
\begin{aligned}
& E=E_{p}^{+}=\frac{|n|}{2}+p, \quad p=1,2, \ldots, \\
& E=E_{p}^{-}=-\frac{|n|}{2}-p, \quad p=1,2, \ldots, \\
& E=E_{0}=\mp \frac{|n|}{2} .
\end{aligned}
$$

Here the upper (lower) sign refers to the BPS (anti-BPS) configuration. The eigenspace with eigenvalue $E$ has degeneracy $2|E|$ and furnishes an irreducible representation of $\mathrm{SU}(2)_{\text {rot }}$.

Comparing the fermion spectrum with the results of [22], we see that the inclusion of the scalar $\chi$ causes dramatic changes in the spectrum of fermions. First, unlike in [22, there are no zero modes. Second, the spectrum is not symmetric with respect to $E \rightarrow-E$.

The absence of zero modes, either in the scalar or in the spinor sector, means that for a fixed magnetic flux the state of lowest energy is unique. We will call it the vacuum state. By construction, it is an (anti-) BPS state, and we would like to determine its quantum numbers. It is clear that the vacuum state is rotationally invariant, so its spin is zero. It is also a flavor singlet. The other quantum numbers of interest are the energy (which is the same as the conformal dimension of the corresponding local operator [22]) and the $\mathrm{U}(1)_{B}$ and $\mathrm{U}(1)_{N}$ charges. Vacuum energy and charge are plagued by normalordering ambiguities, as usual, but as in [22] we can deal with them by requiring the state corresponding to the unit operator (i.e. the vacuum with zero magnetic flux) to have zero energy and charges.

The asymmetry of the fermionic energy spectrum leads to a subtlety in the computation. Suppose we use point-splitting regularization to define vacuum energy and charges. Then one gets different results after renormalization depending on the ordering of operators $\psi$ and $\bar{\psi}$. For example, consider two definitions of the $\mathrm{U}(1)_{N}$ charge

$$
\begin{aligned}
& N(\tau)=\lim _{\beta \rightarrow 0+}\left[\int_{\mathbb{S}^{2}}-\bar{\psi}\left(\tau+\frac{\beta}{2}\right) \sigma_{\tau} \psi\left(\tau-\frac{\beta}{2}\right)-\overline{\tilde{\psi}}\left(\tau+\frac{\beta}{2}\right) \sigma_{\tau} \tilde{\psi}\left(\tau-\frac{\beta}{2}\right)-C(\beta)\right], \\
& N^{\prime}(\tau)=\lim _{\beta \rightarrow 0+}\left[\int_{\mathbb{S}^{2}} \psi\left(\tau+\frac{\beta}{2}\right) \sigma_{\tau} \bar{\psi}\left(\tau-\frac{\beta}{2}\right)+\tilde{\psi}\left(\tau+\frac{\beta}{2}\right) \sigma_{\tau} \overline{\tilde{\psi}}\left(\tau-\frac{\beta}{2}\right)-C^{\prime}(\beta)\right],
\end{aligned}
$$

where $\tau$ is the time coordinate on $\mathbb{S}^{2} \times \mathbb{R}$, and $C(\beta)$ and $C^{\prime}(\beta)$ are c-numbers defined as the $\mathrm{U}(1)_{N}$ charge of the vacuum with $n=0$ regularized by means of appropriate point-splitting. One can easily see that these two definitions are equivalent only if the fermion spectrum is symmetric with respect to zero; otherwise they differ by a c-number which depends on $n$. This ambiguity can be removed by requiring that the regularization procedure preserve charge-conjugation symmetry. This mandates using expressions symmetrized with respect to $\psi$ and $\bar{\psi}$ (and $\tilde{\psi}$ and $\tilde{\psi}$ ). Thus we will define the $\mathrm{U}(1)_{N}$ charge as the average of $N(\tau)$ and $N^{\prime}(\tau)$. The same applies to the $\mathrm{U}(1)_{B}$ charge and the energy operator. 
As an illustration, let us compute the $\mathrm{U}(1)_{N}$ charge of the vacuum for arbitrary $n$. The above definition yields the following regularized $\mathrm{U}(1)_{N}$ charge:

$$
N_{r e g}(\beta)=N_{f} \sum_{E} 2|E| \operatorname{sign}(E) e^{-\beta|E|} .
$$

Here the summation extends over the fermion energy spectrum, and we took into account that $\psi$ and $\tilde{\psi}$ have the same spectrum and $\mathrm{U}(1)_{N}$ charge and contribute equally to $N_{\text {reg }}(\beta)$. The regularized charge of the unit operator is identically zero, since the spectrum is symmetric for $n=0$. For non-zero vortex charge the spectrum is symmetric except for a single eigenvalue $E_{0}$. Thus the renormalized charge is equal to

$$
N_{\text {vac }}= \pm \lim _{\beta \rightarrow 0+} N_{f}|n|= \pm N_{f}|n|,
$$

where the upper (lower) sign refers to the BPS (anti-BPS) state. Since the spectrum of scalars is symmetric, only spinors will contribute to the $\mathrm{U}(1)_{B}$ charge of the vacuum, and an identical argument gives

$$
B_{v a c}=\mp N_{f}|n| .
$$

A similar, but slightly longer, computation gives the vacuum energy:

$$
E=\frac{|n| N_{f}}{2}
$$

This is the same as the scaling dimension of the corresponding monopole operator.

Recall that at large $N_{f}$ the R-charge which is the superpartner of the hamiltonian is given by

$$
R_{\mathrm{IR}}=N+\frac{1}{2} B
$$

It is easy to see from the above results that $E= \pm R_{\mathrm{IR}}$ for our "vacuum" states. This is a satisfying result, since in a unitary $3 \mathrm{~d}$ CFT the scaling dimension of any (anti-) chiral primary must be equal to (minus) its R-charge.

As expected, the energy and the R-charge of the vacuum are of order $N_{f}$. Other states can be obtained by acting on the vacuum with a finite number of creation operators for the charged fields. If the number of creation operators is kept fixed in the limit of large $N_{f}$, then both $E$ and $R_{\mathrm{IR}}$ tend to infinity, with $E-R_{\mathrm{IR}}$ kept finite. Thus the limit we are considering is qualitatively similar to the PP-wave limit of $N=4 d=4$ SYM theory considered in [23]. But since we are taking the number of flavors, rather than the number of colors, to infinity, the physics is rather different. For example, in [23] the combination $R^{2} / N_{c}$ is kept fixed and can be an arbitrary positive real number (it is the effective string coupling in the dual string theory). The analogous quantity in our case is $2 R_{\mathrm{IR}} / N_{f}=|n|$, the vortex charge, which is quantized.

One issue which we have not mentioned yet is gauge-invariance. In order for the operator to be gauge-invariant, the corresponding state must satisfy the Gauss law constraint. In the limit $e \rightarrow \infty$ this is equivalent to requiring that the state be annihilated by the electric charge density operator [22]. For the vacuum state, this is automatic. For excited states, the Gauss law constraint is a non-trivial requirement. 
We have identified above a scalar state on $\mathbb{S}^{2} \times \mathbb{R}$ which is a chiral primary. What about its superpartners? The key point is to realize that the classical field configuration we are considering breaks some of the symmetries of the CFT. In such a situation, one must enlarge the Hilbert space by extra variables ("zero modes") which correspond to the broken generators. In other words, the semi-classical Hilbert space is obtained by tensoring the "naive" Hilbert space by the space of functions on the coset $G / H$, where $G$ is the symmetry group of the theory, and $H$ is the invariance subgroup of the classical configuration. This observation plays an important role in the quantization of solitons. For example, if we are dealing with a soliton in a Poincaré-invariant theory which breaks translational symmetry to nothing, but preserves rotational symmetry, the zero mode Hilbert space is

$$
\operatorname{ISO}(d-1,1) / \operatorname{SO}(d-1,1)=\mathbb{R}^{d-1,1} .
$$

Poincare group acts on the space of functions on $\mathbb{R}^{d-1,1}$ in the usual manner. Furthermore, if a soliton breaks some of supersymmetries, there will be fermionic zero modes, and the bosonic coset must be replaced by an appropriate supercoset.

In our case, the symmetry of theory is described by the $N=2 d=3$ super-Poincare group. ${ }^{3}$ For the BPS state, the invariance subgroup is generated by rotations and the complex supercharge $\bar{Q}_{\alpha}$. Thus the zero mode Hilbert space will consist of functions on the supercoset

$$
\frac{\left\{\mathrm{M}_{i j}, \mathrm{P}_{i}, \mathrm{Q}_{\alpha}, \overline{\mathrm{Q}}_{\alpha}\right\}}{\left\{\mathrm{M}_{i j}, \overline{\mathrm{Q}}_{\alpha}\right\}}
$$

where $\mathrm{M}_{i j}$ and $\mathrm{P}_{i}$ are the rotation and translation generators on $\mathbb{R}^{3}$, respectively, and $\{A, B, \ldots\}$ denotes the super-group with Lie super-algebra spanned by $A, B, \ldots$ Functions on this supercoset are nothing but $N=2 d=3$ chiral superfields [24]. Thus the usual rules of semi-classical quantization lead to the conclusion that the BPS monopole operator is described by a chiral superfield. Similarly, an anti-BPS monopole operator will be described by an anti-chiral superfield. In particular, $N=2$ auxiliary fields are automatically incorporated. (Note that at large $N_{f}$ our monopole operators are not expected to satisfy any closed equation of motion. On the other hand, auxiliary fields can be eliminated only on-shell. This suggests that any description of monopole operators without auxiliary fields would be rather cumbersome.)

\subsection{A comparison with the predictions of $N=2$ mirror symmetry}

As explained above, under mirror symmetry the vortex charge is mapped to $1 / N_{f}$ times the charge which "counts" the number of $q$ 's minus the number of $\tilde{q}$ 's. Thus the obvious gauge-invariant chiral primaries with vortex charge \pm 1 are

$$
V_{+}=q_{1} q_{2} \cdots q_{N_{f}}, \quad V_{-}=\tilde{q}_{1} \tilde{q}_{2} \cdots \tilde{q}_{N_{f}} .
$$

\footnotetext{
${ }^{3}$ We may forget about $\mathrm{U}(1)_{N}, \mathrm{U}(1)_{B}$, and the flavor symmetry, since they are left unbroken by our field configuration. Furthermore, although conformal and superconformal boosts do not preserve our field configuration, they can be ignored, since these symmetry generators cannot be exponentiated to well-defined symmetry transformations on $\mathbb{R}^{3}$.
} 
Using the matching of global symmetries explained above, we see that both $V_{+}$and $V_{-}$ are singlets under $\mathrm{SU}\left(N_{f}\right) \times \mathrm{SU}\left(N_{f}\right)$ flavor symmetry, have $\mathrm{U}(1)_{B}$ charge $-N_{f}$ and $\mathrm{U}(1)_{N}$ charge $N_{f}$. Comparing this with the previous subsection, we see that $V_{+}$has the same quantum numbers as the BPS state with $n=1$ that we have found, while $V_{-}^{\dagger}$ has the same quantum numbers as the anti-BPS state with $n=1$. This agreement provides a non-trivial check of $N=2$ mirror symmetry.

Our computation of the charges was performed in the large- $N_{f}$ limit, but mirror symmetry predicts that the result remains true for $N_{f}$ of order 1 . Can we understand this apparent lack of $1 / N_{f}$ corrections to $\mathrm{U}(1)_{N}$ and $\mathrm{U}(1)_{B}$ charges? The answer is yes: $\mathrm{U}(1)_{N}$ and $\mathrm{U}(1)_{B}$ charges are not corrected at any order in $1 / N_{f}$ expansion because they can be determined by quasi-topological considerations $\left(L^{2}\right.$ index theorem on $\left.\mathbb{S}^{2} \times \mathbb{R}\right)$. This will be discussed in more detail in section 4 .

\section{Monopole operators in $N=4 d=3$ SQED}

\subsection{Review of $N=4$ SQED and $N=4$ mirror symmetry}

$N=4 d=3 \mathrm{SQED}$ is the dimensional reduction of $N=2 d=4$ SQED. The supersymmetry algebra includes two complex spinor supercharges $\mathrm{Q}_{\alpha}^{i}, i=1,2$ and their complex conjugates. In Minkowski signature, the spinor representation is real, so we may also say that we have four real spinor supercharges. If we regard $N=4$ SQED as an $N=2 d=3$ gauge theory, then it contains, besides the fields of $N=2$ SQED, a chiral superfield $\Phi$. This superfield is neutral and together with the $N=2$ vector multiplet $V$ forms an $N=4$ vector multiplet. The chiral superfields $Q_{j}$ and $\tilde{Q}_{j}^{\dagger}$ combine into an $N=4$ hypermultiplet. The action of $N=4$ SQED is the sum of the action of $N=2$ SQED, the usual kinetic term for $\Phi$, and a superpotential term

$$
\int d^{3} x d^{2} \theta \sum_{j=1}^{N_{f}} Q_{j} \Phi \tilde{Q}_{j}+\text { h.c. }
$$

The flavor symmetry of this theory is $\mathrm{SU}\left(N_{f}\right)$. In addition, there is an important Rsymmetry $\mathrm{SU}(2)_{R} \times \mathrm{SU}(2)_{N}$. In the $N=2$ superfield formalism used above, only its maximal torus $\mathrm{U}(1)^{2}$ is manifest. The lowest components of $Q$ and $\tilde{Q}^{\dagger}$ are singlets under $\mathrm{SU}(2)_{N}$ and transform as a doublet under $\mathrm{SU}(2)_{R}$. The complex scalar $\Phi$ in the chiral multiplet and the real scalar $\chi$ in the $N=2$ vector multiplet transform as a triplet of $\mathrm{SU}(2)_{N}$ and are singlets of $\mathrm{SU}(2)_{R}$. The transformation properties of other fields can be inferred from these using the fact that the four real spinor supercharges of $N=4$ SQED transform in the $(2,2)$ representation of $\mathrm{SU}(2)_{R} \times \mathrm{SU}(2)_{N}$.

Although there is a complete symmetry between $\mathrm{SU}(2)_{R}$ and $\mathrm{SU}(2)_{N}$ at the level of superalgebra, the transformation properties of fields do not respect this symmetry. Therefore one can define twisted vector multiplets and twisted hypermultiplets for which the roles of $\mathrm{SU}(2)_{N}$ and $\mathrm{SU}(2)_{R}$ are reversed. $N=4$ SQED contains only "ordinary" vector and hypermultiplets, while its mirror (see below) contains only twisted multiplets. There 
are interesting $N=4$ theories in 3d which include both kinds of multiplets [28, 16], but in this paper we will only consider the traditional ones, which can be obtained by dimensional reduction from $N=2 d=4$ theories.

In order to make contact with our discussion of $N=2 \mathrm{SQED}$, we will denote the global $\mathrm{U}(1)$ symmetry under which $Q$ and $\tilde{Q}$ have charge 1 and $\Phi$ has charge -2 by $\mathrm{U}(1)_{B}$, and we will denote an R-symmetry under which $Q$ and $\tilde{Q}$ are neutral and $\Phi$ has charge 2 by $\mathrm{U}(1)_{N}$. It is easy to see that $\mathrm{U}(1)_{N}$ is a maximal torus of $\mathrm{SU}(2)_{N}$, while the generator of $\mathrm{U}(1)_{B}$ is a linear combination of the generators of $\mathrm{SU}(2)_{N}$ and $\mathrm{SU}(2)_{R}$. The generator of the maximal torus of $\mathrm{SU}(2)_{R}$ can be taken as

$$
R=N+B
$$

$N=4 \mathrm{SQED}$ is free in the UV and flows to an interacting SCFT in the IR. The infrared dimensions of fields in short multiplets of the superconformal algebra are determined by their spin and transformation properties under $\mathrm{SU}(2)_{R} \times \mathrm{SU}(2)_{N}$. This is easily seen in the harmonic superspace formalism, where the compatibility of constraints on the superfields leads to relations between the dimension and the R-spins [24]. For gauge-invariant operators, one can alternatively use arguments based on unitarity (see e.g. [29).

Perhaps the easiest way to work out the relation between the IR dimension and $\mathrm{SU}(2)_{R} \times \mathrm{SU}(2)_{N}$ quantum numbers is to regard $N=4 \mathrm{SQED}$ as a special kind of $N=2$ theory. That is, it is an $N=2$ gauge theory which has, besides a manifest complex supercharge, a non-manifest one. It is easy to see that the combination $N+\frac{1}{2} B$ is the generator of the $\mathrm{U}(1)$ subgroup of $\mathrm{SU}(2)_{N} \times \mathrm{SU}(2)_{R}$ with respect to which the manifest supercharge has charge 1 , while the non-manifest supercharge has charge 0 . In the IR limit, the corresponding current is in the same multiplet as the stress-energy tensor (because all $\mathrm{SU}(2)_{R} \times \mathrm{SU}(2)_{N}$ currents are), and therefore the dimension of chiral primary states must be equal to their charges with respect to $N+\frac{1}{2} B$. (Note that in the case of $N=2$ SQED this was true only in the large- $N_{f}$ limit.) In particular, the IR dimensions of $Q_{j}$ and $\tilde{Q}_{j}$ are $1 / 2$, and the IR dimension of $\Phi$ and $\chi$ is 1 .

According to [6], the mirror theory for $N=4$ SQED is a (twisted) $N=4 d=3$ gauge theory with gauge group $\mathrm{U}(1)^{N_{f}} / \mathrm{U}(1)_{\operatorname{diag}}$ and $N_{f}$ (twisted) hypermultiplets $\left(q_{j}, \tilde{q}_{j}\right)$. The matter multiplets transform under the gauge group as follows:

$$
q_{j} \rightarrow q_{j} e^{i\left(\alpha_{j}-\alpha_{j-1}\right)}, \quad \tilde{q}_{j} \rightarrow \tilde{q}_{j} e^{-i\left(\alpha_{j}-\alpha_{j-1}\right)}, \quad j=1, \ldots, N_{f},
$$

where we set $\alpha_{0}=\alpha_{N_{f}}$. The action of the mirror theory is

$$
\begin{aligned}
S_{\text {dual }}=\int & d^{3} x d^{4} \theta \sum_{j=1}^{N_{f}}\left\{\frac{1}{e^{2}} \Sigma_{j}^{\dagger} \Sigma_{j}+\frac{1}{e^{2}} S_{j}^{\dagger} S_{j}+q_{j}^{\dagger} e^{2 V_{j}-2 V_{j-1}} q_{j}+\tilde{q}_{j}^{\dagger} e^{-2 V_{j}+2 V_{j-1}} \tilde{q}_{j}\right\}+ \\
& +\left(\int d^{3} x d^{2} \theta \sum_{j=1}^{N_{f}} q_{j} \tilde{q}_{j}\left(S_{j}-S_{j-1}\right)+\text { h.c. }\right)
\end{aligned}
$$


Here $N=2$ vector multiplets $V_{j}$ satisfy the constraints eq. (2.1), $N=2$ chiral multiplets $S_{j}$ satisfy similar constraints

$$
S_{0}=S_{N_{f}}, \quad \sum_{j=1}^{N_{f}} S_{j}=0,
$$

and each pair $\left(V_{j}, S_{j}\right)$ forms a (twisted) $N=4$ vector multiplet.

The matching of global symmetries goes as follows. The R-symmetries are trivially identified. The vortex current of $N=4 \mathrm{SQED}$ is mapped to $1 / N_{f}$ times the Noether current corresponding to the following global U(1) symmetry:

$$
q_{j} \rightarrow e^{i \alpha} q_{j}, \quad \tilde{q}_{j} \rightarrow e^{-i \alpha} \tilde{q}_{j}, \quad j=1, \ldots, N_{f} .
$$

The currents corresponding to the maximal torus of $\mathrm{SU}\left(N_{f}\right)$ flavor symmetry of $N=4$ SQED are mapped to the vortex currents

$$
2 \pi J_{j}=* F_{j}, \quad j=1, \ldots, N_{f}, \quad \sum_{j=1}^{N_{f}} J_{j}=0,
$$

where $F_{j}$ is the field-strength of the $j^{\text {th }}$ gauge field. The mapping of the rest of $\operatorname{SU}\left(N_{f}\right)$ currents is not well understood.

\subsection{Monopole operators in $N=4$ SQED at large $N_{f}$}

To begin with, we can regard $N=4 \mathrm{SQED}$ as a rather special $N=2$ gauge theory, and look for BPS and anti-BPS monopole operators in this theory. This amounts to focusing on a particular $N=2$ subalgebra of the $N=4$ superalgebra. Different choices of an $N=2$ subalgebra are all related by an $\mathrm{SU}(2)_{N}$ transformation, so we do not loose anything by doing this.

From this point of view, our problem is almost exactly the same as in the case of $N=2$ SQED. The only difference between the two is the presence of the chiral superfield $\Phi$. But in the large $N_{f}$ limit it becomes non-dynamical, and the $N=2$ BPS condition requires the background value of $\Phi$ to be zero. This implies that the radial quantization of the matter fields $Q_{j}, \tilde{Q}_{j}$ proceeds in exactly the same way as in the $N=2$ case and yields the same answer for the spectrum and properties of BPS and anti-BPS states. Namely, for any vortex charge $n$ we have a single BPS and a single anti-BPS states, with charges

$$
N= \pm|n| N_{f}, \quad B=\mp|n| N_{f},
$$

and energy $E=|n| N_{f} / 2$.

An interesting new element in the $N=4$ case is the way short multiplets of $N=2$ superconformal symmetry fit into a short multiplet of $N=4$ superconformal symmetry. Recall that we have made a certain choice of $N=2$ subalgebra of the $N=4$ superalgebra. This choice is preserved by the $\mathrm{U}(1)_{N}$ symmetry, but not by the $\mathrm{SU}(2)_{N}$ symmetry. Thus we have an $\mathrm{SU}(2) / \mathrm{U}(1) \simeq \mathbb{C P}^{1}$ worth of BPS conditions. Applying an $\mathrm{SU}(2)_{N}$ rotation to 
the BPS state found above, we obtain a half-BPS state for every point on $\mathbb{C P}^{1}$. These halfBPS states fit into a line bundle $\mathcal{L}$ over $\mathbb{C P}^{1}$. Similarly, applying $\mathrm{SU}(2)_{N}$ transformations to the anti-BPS state, we obtain another line bundle on $\mathbb{C P}^{1}$ which is obviously the complex conjugate of $\mathcal{L}$.

The $\mathbb{C P}^{1}$ which parametrizes different choices of the $N=2$ subalgebra has a very clear meaning in the large $N_{f}$ limit. Namely, we chose the scalar background on $\mathbb{S}^{2} \times \mathbb{R}$ to be $\Phi=0, \chi=n / 2$, but obviously any $\mathrm{SU}(2)_{N}$ transform of this is also a half-BPS configuration. The manifold of possible scalar backgrounds is a 2 -sphere given by

$$
|\Phi|^{2}+\chi^{2}=\left(\frac{n}{2}\right)^{2}
$$

The BPS state we are interested in is the Fock vacuum of charged matter fields on $\mathbb{S}^{2} \times \mathbb{R}$ in a fixed background. As we vary the background values of $\Phi$ and $\chi$, we obtain a bundle of Fock vacua on $\mathbb{S}^{2} \sim \mathbb{C P}^{1}$. This bundle can be non-trivial because of Berry's phase [30, 31.

Now we can easily see how $N=4$ superconformal symmetry is realized in our formalism. As argued above, we need to enlarge our Hilbert space by the Hilbert space of zero modes, which arise because the classical background breaks some of the symmetries of the theory. Compared to the $N=2$ case, we have additional bosonic zero modes coming from the breaking of R-symmetry from $\mathrm{SU}(2)_{N}$ down to $\mathrm{U}(1)_{N}$. Thus our fields will depend on coordinates on $\mathbb{R}^{3} \times \mathbb{C P}^{1}$. As for fermionic zero modes, in the BPS case they are generated by a complex spinor supercharge which depends on the coordinates on $\mathbb{C P}^{1}$ as follows:

$$
\mathrm{Q}_{\alpha}=\sum_{i=1,2} u_{i} \mathrm{Q}_{\alpha}^{i}
$$

Here $u_{1}, u_{2} \in \mathbb{C}$ are homogeneous coordinates on $\mathbb{C P}^{1}$, and $\mathrm{Q}_{\alpha}^{i}, i=1,2$ are a pair of complex spinor supercharges which transform as a doublet of $\mathrm{SU}(2)_{N}$. Therefore monopole operators will be described by "functions" on the supermanifold

$$
S\left(\mathbb{R}^{3}\right) \otimes \mathcal{O}(1),
$$

where $S\left(\mathbb{R}^{3}\right)$ is the trivial spinor bundle on $\mathbb{R}^{3}$ (with fiber coordinates regarded as Grassmann-odd), while $\mathcal{O}(1)$ is the tautological line bundle on $\mathbb{C P}^{1}$. We put the word "functions" in quotes, because, as explained above, we may need to consider sections of non-trivial line bundles on $\mathbb{C P}^{1}$ instead of functions.

This supermanifold is known as the analytic superspace [25, 26, 24] (see also [27, section 3]). It is a chiral version of the so-called harmonic superspace. It is well known that "functions" on the analytic superspace (analytic superfields) furnish short representations of the superconformal algebra with eight supercharges [24]. We conclude that in the large$N_{f}$ limit BPS monopole operators are described by $N=4 d=3$ analytic superfields. Needless to say, anti-BPS monopole operators are described by anti-analytic superfields which are complex-conjugates of the analytic ones.

It remains to pin down the topology of the bundle $\mathcal{L}$ over $\mathbb{C P}^{1}$. Since this is a line bundle, its topology is completely characterized by the first Chern class. A "cheap" way to find the Chern class is to note that the scaling dimension of an analytic superfield (more 
precisely, of its scalar component) is equal to half the Chern number of the corresponding line bundle. (The Chern number is the value of the first Chern class on the fundamental homology class of $\mathbb{C} \mathbb{P}^{1}$.) This follows from the way superconformal algebra is represented on analytic superfields [24]. We already know the dimension of our BPS state, and therefore infer that the Chern number of $\mathcal{L}$ is equal to $N_{f}|n|$.

We can also determine the Chern number directly, by computing the curvature of the Berry connection for the bundle of Fock vacua. In the present case, the computation is almost trivial, since the hamiltonians at different points of $\mathbb{C P}^{1}$ are related by an $\mathrm{SU}(2)_{N}$ transformation. In particular, it is sufficient to compute the curvature at any point on $\mathbb{C P}^{1}$. For example, we can identify $\mathbb{C P}^{1}$ with a unit sphere in $\mathbb{R}^{3}$ with coordinates $(x, y, z)$ and compute the curvature at the "North Pole," which has euclidean coordinates $(0,0,1)$. (The abstract coordinates $(x, y, z)$ can be identified with $(\operatorname{Re} \Phi, \operatorname{Im} \Phi, \chi)$.) Using $\operatorname{SU}(2)_{N}$ invariance, we easily see that the Fock vacuum at the point $(x, y, z)$ with $z \simeq 1, x, y \ll 1$ is given by

$$
|x, y, z\rangle=\exp \left(i\left(\frac{x}{z} N_{x}-\frac{y}{z} N_{y}\right)+O\left(x^{2}+y^{2}\right)\right)|0,0,1\rangle .
$$

Here $N_{x}$ and $N_{y}$ are the generators of $\mathrm{SU}(2)_{N}$ rotations about $x$ and $y$ axes. Therefore the curvature of the Berry connection at the point $(0,0,1)$ is

$$
\begin{aligned}
\mathcal{F} & =i(d|x, y, z\rangle, \wedge d|x, y, z\rangle)=i d x \wedge d y\left\langle 0,0,1\left|\left[N_{y}, N_{x}\right]\right| 0,0,1\right\rangle \\
& =d x \wedge d y\left\langle 0,0,1\left|N_{z}\right| 0,0,1\right\rangle .
\end{aligned}
$$

Now we recall that the vacuum at $(0,0,1)$ is an eigenstate of $N_{z}$ with eigenvalue $\pm N_{f}|n| / 2$ (one needs to remember that $N=2 N_{z}$ ). Taking into account that $\mathcal{F}$ is an $\mathrm{SU}(2)_{N}$-invariant 2-form on $\mathbb{C P}^{1}$, we conclude that it is given by

$$
\mathcal{F}= \pm \frac{1}{2} N_{f}|n| \Omega
$$

where $\Omega$ is the volume form on the unit 2 -sphere. It follows that the Chern number of the Fock vacuum bundle is

$$
c_{1}=\frac{1}{2 \pi} \int_{S^{2}} \mathcal{F}= \pm N_{f}|n|
$$

where the upper (lower) sign refers to $\mathcal{L}\left(\right.$ resp. $\left.\mathcal{L}^{*}\right)$. The result agrees with the indirect argument given above.

\subsection{A comparison with the predictions of $N=4$ mirror symmetry}

Chiral primaries in the mirror theory with vortex number \pm 1 are exactly the same as in the $N=2$ case, i.e.

$$
V_{+}=q_{1} q_{2} \cdots q_{N_{f}}, \quad V_{-}=\tilde{q}_{1} \tilde{q}_{2} \cdots \tilde{q}_{N_{f}} .
$$

Their $\mathrm{U}(1)_{N}$ and $\mathrm{U}(1)_{B}$ quantum numbers match those computed in the original theory using radial quantization and large- $N_{f}$ expansion. This provides a check of $N=4$ mirror symmetry at the origin of the moduli space. We can also translate this into the language of analytic superfields. Then a hypermultiplet $\left(q_{j}, \tilde{q}_{j}^{\dagger}\right)$ is described by an analytic superfield 
$\mathrm{q}_{j}$ whose Chern number is 1 (in the notation of [24], it would be written as $q_{j}^{+}$, where a single + superscript refers to the unit Chern number). The analytic superfield which is gauge-invariant and carries vortex charge 1 is given by

$$
\mathrm{q}_{1} \mathrm{q}_{2} \ldots \mathrm{q}_{N_{f}} \text {. }
$$

It has Chern number $N_{f}$, and in the notation of 24] it would have $N_{f}$ superscripts. This field corresponds to the BPS multiplet constructed in the previous section, while its complex conjugate corresponds to the anti-BPS multiplet.

Mirror symmetry also predicts a certain interesting relation in the chiral ring of the IR limit of $N=4$ SQED. Consider the product of $V_{+}$and $V_{-}$:

$$
V_{+} V_{-}=\left(q_{1} \tilde{q}_{1}\right)\left(q_{2} \tilde{q}_{2}\right) \ldots\left(q_{N_{f}} \tilde{q}_{N_{f}}\right) .
$$

Using the equation of motion for $S_{j}$, it is easy to see that the operators $\left(q_{j} \tilde{q}_{j}\right)$ for different $j$ are equal modulo descendants. Furthermore, mirror symmetry maps any of these operators to $\Phi$ modulo descendants [12. Thus we infer that modulo descendants we have a relation in the chiral ring:

$$
V_{+} V_{-} \sim \Phi^{N_{f}} .
$$

Can we understand this relation in terms of $N=4$ SQED? Indeed we can!

To begin with, it is easy to see that the operator $\Phi^{N_{f}}$ is the only chiral operator whose quantum numbers match those of $V_{+} V_{-}$and which could appear in the OPE of $V_{+}$and $V_{-}$. Thus it is sufficient to demonstrate that it appears with a non-zero coefficient. To this end, we need to compute the 3-point function of $V_{+}, V_{-}$, and $\left(\Phi^{\dagger}\right)^{N_{f}}$. In the radial quantization approach, we need to show that the matrix element

$$
\left\langle V_{-}^{\dagger}\left|\left(\Phi^{\dagger}\right)^{N_{f}}\right| V_{+}\right\rangle
$$

is non-zero.

Now we recall that the state corresponding to $V_{+}$has magnetic flux +1 and scalar VEV $\chi=1 / 2$, while the state corresponding to $V_{-}$has magnetic flux -1 and $\chi=-1 / 2$. hermitean conjugation reverses the sign of the magnetic flux and leaves the VEV of $\chi$ unchanged. It follows that the path integral which computes the matrix element of any operator between $\left\langle V_{-}^{\dagger}\right|$ and $\left|V_{+}\right\rangle$must be performed over field configurations such that the magnetic flux is equal to 1 , while the scalar $\chi$ asymptotes to $1 / 2$ at $\tau=-\infty$ and $-1 / 2$ at $\tau=+\infty$. Thus we are dealing with a kink on $\mathbb{S}^{2} \times \mathbb{R}$.

Next, we note that the Dirac operator on $\mathbb{S}^{2} \times \mathbb{R}$ coupled to such a background may very well have normalizable zero modes. If this is the case, then in order to get a non-zero matrix element one needs to insert an operator which has the right quantum numbers to absorb the zero modes. For example, one can insert a product of all fermionic fields which possess a zero mode. Another possibility, which is more relevant for us, is to insert some bosonic fields which interact with fermions and can absorb the zero modes. In our case, the action contains a complex scalar $\Phi$ which has Yukawa interactions of the form

$$
\int d^{3} x \Phi \sum_{j=1}^{N_{f}} \psi_{j} \tilde{\psi}_{j} .
$$


Thus if each $\psi$ and each $\tilde{\psi}$ has a single normalizable zero mode, then we can get a non-zero result for the matrix element if we insert precisely $N_{f}$ powers of $\Phi^{\dagger}$.

To complete the argument it remains to show that the Dirac operator for both $\psi$ and $\tilde{\psi}$ has a single zero mode. The Atiyah-Patodi-Singer theorem says in this case that the $L^{2}$ index of the Dirac operator is

$$
\operatorname{ind}(D)=\frac{1}{2}\left(\eta\left(H_{-}\right)-\eta\left(H_{+}\right)\right),
$$

where $\eta\left(H_{ \pm}\right)$denotes the $\eta$-invariant of the asymptotic Dirac hamiltonian at $\tau \rightarrow \pm \infty$. We also made use of the fact that neither $H_{+}$nor $H_{-}$have zero modes (see section 2). Now we recall that we have computed the $\eta$-invariants already: according to eq. (2.2), $\eta\left(H_{-}\right)$ and $\eta\left(H_{+}\right)$coincide with the $\mathrm{U}(1)_{N}$ charges of the BPS and anti-BPS vacua, respectively, divided by $N_{f}$. This implies that the index of the Dirac operator is equal to 1 , for both $\psi$ and $\tilde{\psi}$, and therefore both $\psi$ and $\tilde{\psi}$ have a single zero mode.

\section{Beyond the large- $N_{f}$ limit}

\subsection{Non-renormalization theorems for the anomalous charges}

We have seen that mirror symmetry makes certain predictions about the quantum numbers of BPS monopole operators, and that our large- $N_{f}$ computations confirm these predictions. But mirror symmetry also suggests that large- $N_{f}$ results for $\mathrm{U}(1)_{B}$ and $\mathrm{U}(1)_{N}$ charges remain valid for all $N_{f}$, all the way down to $N_{f}=1$. In this subsection we provide an explanation for this without appealing to mirror symmetry. We show that the values of $\mathrm{U}(1)_{N}$ and $\mathrm{U}(1)_{B}$ charges for monopole operators are fixed by the $L^{2}$ index theorem for the Dirac operator on $\mathbb{S}^{2} \times \mathbb{R}$ and therefore cannot receive $1 / N_{f}$ corrections.

The argument is very simple. For concreteness, consider the monopole operators $V_{ \pm}$ which have vortex charge $n= \pm 1$. These operators are related by charge conjugation and thus have the same $\mathrm{U}(1)_{N}$ charge, which we denote $N_{V}$. To determine $N_{V}$, we need to consider the transition amplitude on $\mathbb{S}^{2} \times \mathbb{R}$ from the state corresponding to $V_{+}$to the state corresponding to $V_{-}^{\dagger}$ : if it violates the $\mathrm{U}(1)_{N}$ charge by $m$, then $N_{V}=-m / 2$. Since $\psi$ and $\tilde{\psi}$ have $N=-1$, the charge is violated by $-2 N_{f}$ times the index of the Dirac operator on $\mathbb{S}^{2} \times \mathbb{R}$. The index of the Dirac operator in the present case has only boundary contributions ( $\eta$-invariants), which depend on the asymptotics of the gauge field and the scalar $\chi$. When these asymptotics are given by the large- $N_{f}$ saddle points, the index was evaluated in section 3 with the result ind $(D)=1$. Furthermore, in the large- $N_{f}$ expansion fluctuations about the saddle point are treated using perturbation theory. Hence to all orders in $1 / N_{f}$ expansion the transition amplitude from $V_{+}$to $V_{-}^{\dagger}$ will violate $\mathrm{U}(1)_{N}$ charge by $-2 N_{f}$. This implies that the $\mathrm{U}(1)_{N}$ charge of $V_{ \pm}$is equal to $N_{f}$ to all orders in $1 / N_{f}$ expansion. An identical argument can be made for $\mathrm{U}(1)_{B}$.

One may ask if it is possible to dispense with the crutch of $1 / N_{f}$ expansion altogether. Naively, there is no problem: we consider the path integral for $N=4$ or $N=2$ SQED with $e=\infty$ and use the APS index theorem to infer the charges of $V_{ \pm}$. However, this argument is only formal, because we do not know how to make sense of this path integral without 
using $1 / N_{f}$ expansion. In particular, this leads to difficulties with the evaluation of the index: we cannot compute the $\eta$-invariants without knowing the precise asymptotic form of the background, but the asymptotic conditions put constraints only on the total magnetic flux through $\mathbb{S}^{2}$ and the average value of $\chi$ at $\tau= \pm \infty$. (We remind that the $L^{2}$-index of a Dirac operator on a non-compact manifold is only a quasi-topological quantity, which can change if the asymptotic behavior of the fields is changed.) The index has a definite value only if we choose some particular asymptotics for the gauge field and $\chi$.

\subsection{A derivation of the basic $N=4$ mirror symmetry}

It is plausible that the point $N_{f}=1$ is within the radius of convergence of $1 / N_{f}$ expansion. Singularities in an expansion parameter usually signal some sort of phase transition, and in the case of $N=4$ SQED we do not expect any drastic change of behavior as one decreases $N_{f}$.

With this assumption, we can prove the basic example of $N=4$ mirror symmetry, namely, that the IR limit of $N=4$ SQED with $N_{f}=1$ is dual to the theory of a free twisted hypermultiplet. The proof is quite straightforward. As explained above, the $\mathrm{U}(1)_{N}$ charge of the chiral field $V_{ \pm}$is equal to $N_{f}$ to all orders in $1 / N_{f}$ expansion, while its $\mathrm{U}(1)_{B}$ charge is equal to $-N_{f}$. This implies that the IR dimension of $V_{+}$is equal to $N_{f} / 2$ to all orders in $1 / N_{f}$ expansion (see section 3). Assuming that $1 / N_{f}$ expansion converges at $N_{f}=1$, this implies that for $N_{f}=1$ the IR dimension of $V_{ \pm}$is $1 / 2$. In a unitary 3d CFT, a scalar of dimension $1 / 2$ must be free [29]. Then, by virtue of supersymmetry, the $N=2$ superfields $V_{ \pm}$are free chiral superfields with $N=1$ and $B=-1$, or, equivalently, the pair $\left(V_{+}, V_{-}^{\dagger}\right)$ is a free twisted hypermultiplet.

The above argument shows that the IR limit of $N=4$ SQED contains a free sector generated by the action of free fields $V_{ \pm}$on the vacuum. But this sector also contains all the states generated by $\Phi$ and its superpartners. Indeed, the product of $V_{+}$and $V_{-}$is a chiral field which has zero vortex charge and $N=2, B=-2$. It is easy to see that the only such field is $\Phi$. In addition, since $V_{+}$and $V_{-}$are independent free fields, their product is non-zero. Thus we must have $V_{+} V_{-} \sim \Phi$ (we have seen above how a more general relation eq. (3.1) can be demonstrated in the large- $N_{f}$ limit). We conclude that the sector of the IR limit of $N=4$ SQED generated by $\Phi$ and its superpartners is contained in the charge- 0 sector of the theory of a free twisted hypermultiplet. This is precisely the statement of mirror symmetry in this particular case.

\section{Discussion of results and open problems}

In this paper we showed that many predictions of three-dimensional mirror symmetry can be verified directly at the origin of the moduli space, where the IR theory is an interacting SCFT. The main idea was that the $e \rightarrow \infty$ limit of $3 \mathrm{~d}$ gauge theories can be defined in the continuum using large- $N_{f}$ expansion, and then vortex-creating operators can be rigorously defined as well. Focusing on vortex-creating operators in short representations of the superconformal algebra, we showed that their transformation laws under various symmetries are determined by index theorems on $\mathbb{S}^{2} \times \mathbb{R}$ and therefore are not corrected 
at any order in $1 / N_{f}$. In the $N=4$ case, this allowed us to determine the exact scaling dimensions of vortex-creating operators to all orders in $1 / N_{f}$ expansion. If we assume that $N_{f}=1$ is within the convergence radius of this expansion, we can prove the basic $N=4$ mirror symmetry, which says that a certain large sector of the IR limit of $N=4 N_{f}=1$ SQED can be described in terms of a free twisted hypermultiplet.

We feel that these results go some way towards making the $3 \mathrm{~d}$ mirror symmetry conjecture into a theorem (on the physical level of rigor). On the other hand, much yet remains to be done before one can claim that one understands $3 \mathrm{~d}$ mirror symmetry. First, it would be desirable to construct monopole operators directly, using hamiltonian formalism on $\mathbb{R}^{3}$, rather than by identifying the corresponding states on $\mathbb{S}^{2} \times \mathbb{R}$. Mandelstam's construction of soliton-creating operators in the sine-Gordon theory [2] serves as a model in this respect. Second, it would be interesting to find the mirror of more complicated observables in $N=4$ SQED. Third, mirror symmetry predicts that many $3 \mathrm{~d}$ gauge theories have "accidental" symmetries in the infrared limit [6, 14]. It appears possible to understand the origin of these symmetries using the methods of this paper. Fourth, for $N_{f}>1$ the mirror theory of $N=4$ SQED is a gauge theory, and one would like to have a conceptual understanding of the origin of the dual gauge group. Although all abelian mirror pairs can be derived for the "basic" one, the derivation is rather formal and does not shed much light on this question.

More ambitiously, we would like to extend the approach of this paper to non-abelian gauge theories and non-abelian 3d mirror symmetry. It seems that a pre-requisite for this is the ability to construct operators which are not invariant with respect to the dual gauge group out of the original variables (i.e. construct operators representing "dual electrons" or "dual quarks.") This problem is also the major stumbling block for our understanding of $4 \mathrm{~d}$ dualities, and we hope that studying $3 \mathrm{~d}$ mirror symmetry will eventually lead to a progress in proving $4 \mathrm{~d}$ dualities.

\section{A. Radial quantization of $N=2$ SQED}

We start with the lagrangian of $N=1 d=4$ SQED in the conventions of Wess and Bagger [32] and perform a Wick rotation to euclidean signature:

$$
\mathcal{L}_{\mathbb{R}^{4}}=-\left.\mathcal{L}_{\mathbb{R}^{1,3}}\right|_{x^{0}=-i t},\left.\quad V_{0}\right|_{\mathbb{R}^{1,3}}=\left.i \chi\right|_{\mathbb{R}^{4}},
$$

where $V_{0}$ is the time-like coordinate of the $\mathrm{U}(1)$ connection. Then we require that all fields be independent of the euclidean time $t$. This procedure gives the action density for $N=2$ $d=3$ SQED on euclidean $\mathbb{R}^{3}$ :

$$
\begin{aligned}
\mathcal{L}= & i \bar{\psi} \vec{\sigma}(\vec{\nabla}+i \vec{V}) \psi+i \chi \bar{\psi} \psi+i \overline{\tilde{\psi}} \vec{\sigma}(\vec{\nabla}-i \vec{V}) \tilde{\psi}-i \chi \overline{\tilde{\psi}} \tilde{\psi}+\chi^{2}\left(A A^{*}+\tilde{A} \tilde{A}^{*}\right)+ \\
& +([\vec{\nabla}+i \vec{V}] A)\left([\vec{\nabla}-i \vec{V}] A^{*}\right)+([\vec{\nabla}-i \vec{V}] \tilde{A})\left([\vec{\nabla}+i \vec{V}] \tilde{A}^{*}\right)- \\
& -D\left(A A^{*}-\tilde{A} \tilde{A}^{*}\right)+i \sqrt{2}\left(A \bar{\psi} \bar{\lambda}-A^{*} \psi \lambda-\tilde{A} \overline{\tilde{\psi}} \bar{\lambda}+\tilde{A}^{*} \tilde{\psi} \lambda\right)+O\left(\frac{1}{e^{2}}\right)
\end{aligned}
$$


In the infrared limit $e \rightarrow \infty$ the kinetic terms for the vector multiplet can be ignored. Note also that in the $e \rightarrow \infty$ limit the equation of motion for $D$ enforces the vanishing of D-terms.

To go from $\mathbb{R}^{3}$ to $\mathbb{S}^{2} \times \mathbb{R}$, we perform a Weyl rescaling of the euclidean metric $d s^{2}=$ $d r^{2}+r^{2} d \Omega^{2}$ by a factor $1 / r^{2}$. If we set $r=e^{\tau}$, then $\tau$ is an affine parameter on $\mathbb{R}$. The component fields of $Q$ must be rescaled as follows:

$$
\psi \rightarrow e^{-\tau} \psi, \quad \bar{\psi} \rightarrow e^{-\tau} \bar{\psi}, \quad A \rightarrow e^{-\frac{\tau}{2}} A, \quad A^{*} \rightarrow e^{-\frac{\tau}{2}} A^{*} .
$$

The component fields of $\tilde{Q}$ transform in a similar way. The bosonic fields in the vector multiplet transform as follows:

$$
\chi \rightarrow e^{-\tau} \chi, \quad \vec{V} \rightarrow \vec{V} .
$$

To find the one-particle energy spectrum for charged fields, we use the procedure and notations of 22]. The lagrangian for $\psi$ and $\bar{\psi}$ in the background of the (anti-) BPS monopole on $\mathbb{R}^{3}$ has the following form

$$
\mathcal{L}[\psi, \bar{\psi}]_{\mathbb{S}^{2} \times \mathbb{R}}=i \bar{\psi} \sigma_{r}\left[\frac{\partial}{\partial \tau}-\left(\vec{J}^{2}-\vec{L}^{2}+\frac{1}{4}\right)-q \sigma_{r} \mp q \sigma_{r}\right] \psi
$$

where $q=-e g=-n / 2$, and the upper (lower) sign corresponds to a BPS (anti-BPS) monopole. A solution with energy $E$ has the form $\psi \sim e^{-E \tau}, \bar{\psi} \sim e^{E \tau}$. The above lagrangian is the same as in [22], except for the last term in brackets. We will not repeat the diagonalization procedure and simply quote the resulting energy spectrum for $\psi$ and $\tilde{\psi}$ :

$$
-\frac{|n|}{2}-p, \quad \mp \frac{|n|}{2}, \quad \frac{|n|}{2}+p,
$$

where $p=1,2, \ldots$ Each energy-level has $\operatorname{spin} j=|E|-1 / 2$ and degeneracy $2 j+1=2|E|$.

The lagrangian for $A, A^{*}$ is

$$
\mathcal{L}\left[A, A^{*}\right]_{\mathbb{S}^{2} \times \mathbb{R}}=\left[\left(\vec{\nabla}_{a}+i \vec{V}_{a}\right) A\right]\left[\left(\vec{\nabla}_{b}-i \vec{V}_{b}\right) A^{*}\right] g^{a b}+\frac{1}{4} A A^{*}+\chi^{2} A A^{*} .
$$

The equation of motion for $A$ has the from

$$
\frac{d^{2}}{d \tau^{2}} A=\left(\vec{L}^{2}+\frac{1}{4}\right) A
$$

where $\vec{L}$ is the generalized angular momentum defined in [33]. Using the known spectrum of $\vec{L}^{2}$, we easily find the one-particle energy spectrum for $A$ and $\tilde{A}$ :

$$
-\frac{|n|-1}{2}-p, \quad \frac{|n|-1}{2}+p, \quad p=1,2, \ldots
$$

The degeneracy of each eigenvalue is again $2|E|$, and each eigenspace is an irreducible representation of the rotation group. 


\section{Acknowledgments}

We would like to thank Jim Gates, Takuya Okuda, Hiroshi Ooguri, John Preskill, and Mark Wise for discussions. A.K. is also grateful to Matt Strassler for numerous conversations during the years of 1998 and 1999 which contributed to the genesis of this paper. This work was supported in part by a DOE grant DE-FG03-92-ER40701.

\section{References}

[1] S.R. Coleman, Quantum Sine-Gordon equation as the massive thirring model, Phys. Rev. D $11(1975) 2088$.

[2] S. Mandelstam, Soliton operators for the quantized Sine-Gordon equation, Phys. Rev. D 11 (1975) 3026.

[3] C. Montonen and D.I. Olive, Magnetic monopoles as gauge particles?, Phys. Lett. B 72 (1977) 117.

[4] E. Witten and D.I. Olive, Supersymmetry algebras that include topological charges, Phys. Lett. B 78 (1978) 97.

[5] H. Osborn, Topological charges for $N=4$ supersymmetric gauge theories and monopoles of spin 1, Phys. Lett. B 83 (1979) 321.

[6] K.A. Intriligator and N. Seiberg, Mirror symmetry in three dimensional gauge theories, Phys. Lett. B 387 (1996) 513 hep-th/9607207.

[7] J. de Boer, K. Hori, H. Ooguri and Y. Oz, Mirror symmetry in three-dimensional gauge theories, quivers and D-branes, Nucl. Phys. B 493 (1997) 101 hep-th/9611063.

[8] M. Porrati and A. Zaffaroni, M-theory origin of mirror symmetry in three dimensional gauge theories, Nucl. Phys. B 490 (1997) 107 hep-th/9611201.

[9] A. Hanany and E. Witten, Type-IIB superstrings, BPS monopoles and three-dimensional gauge dynamics, Nucl. Phys. B 492 (1997) 152 hep-th/9611230.

[10] J. de Boer, K. Hori, H. Ooguri, Y. Oz and Z. Yin, Mirror symmetry in three-dimensional gauge theories, $\mathrm{SL}(2, \mathbb{Z})$ and D-brane moduli spaces, Nucl. Phys. B 493 (1997) 148 hep-th/9612131.

[11] J. de Boer, K. Hori and Y. Oz, Dynamics of $N=2$ supersymmetric gauge theories in three dimensions, Nucl. Phys. B 500 (1997) 163 hep-th/9703100.

[12] O. Aharony, A. Hanany, K.A. Intriligator, N. Seiberg and M.J. Strassler, Aspects of $N=2$ supersymmetric gauge theories in three dimensions, Nucl. Phys. B 499 (1997) 67 hep-th/9703110.

[13] J. de Boer, K. Hori, Y. Oz and Z. Yin, Branes and mirror symmetry in $N=2$ supersymmetric gauge theories in three dimensions, Nucl. Phys. B 502 (1997) 107 hep-th/9702154.

[14] A. Kapustin, $D_{n}$ quivers from branes, J. High Energy Phys. 12 (1998) 015 hep-th/9806238.

[15] T. Kitao, K. Ohta and N. Ohta, Three-dimensional gauge dynamics from brane configurations with (p,q)-fivebrane, Nucl. Phys. B 539 (1999) 79 hep-th/9808111. 
[16] A. Kapustin and M.J. Strassler, On mirror symmetry in three dimensional abelian gauge theories, J. High Energy Phys. 04 (1999) 021 hep-th/9902033.

[17] M. Gremm and E. Katz, Mirror symmetry for $N=1$ QED in three dimensions, J. High Energy Phys. 02 (2000) 008 hep-th/9906020.

[18] N. Dorey and D. Tong, Mirror symmetry and toric geometry in three dimensional gauge theories, J. High Energy Phys. 05 (2000) 018 hep-th/9911094.

[19] D. Tong, Dynamics of $N=2$ supersymmetric Chern-Simons theories, J. High Energy Phys. 07 (2000) 019 hep-th/0005186.

[20] M. Aganagic, K. Hori, A. Karch and D. Tong, Mirror symmetry in 2+1 and $1+1$ dimensions, J. High Energy Phys. 07 (2001) 022 hep-th/0105075.

[21] S. Gukov and D. Tong, D-brane probes of special holonomy manifolds and dynamics of $N=1$ three-dimensional gauge theories, J. High Energy Phys. 04 (2002) 050 hep-th/0202126.

[22] V. Borokhov, A. Kapustin and X.-k. Wu, Topological disorder operators in three-dimensional conformal field theory, J. High Energy Phys. 11 (2002) 049 hep-th/0206054.

[23] D. Berenstein, J.M. Maldacena and H. Nastase, Strings in flat space and PP waves from $N=4$ super Yang-Mills, J. High Energy Phys. 04 (2002) 013 hep-th/0202021.

[24] A.S. Galperin, E.A. Ivanov, V.I. Ogievetsky and E.S. Sokatchev, Harmonic superspace, Cambridge, UK: Univ. Pr. 2001.

[25] A.Galperin, E. Ivanov, V. Ogievetsky, E. Sokatchev, Harmonic superspace: key to $N=2$ supersymmetry theories, Sov. Phys. JETP Lett. 40 (1984) 912.

[26] A. Galperin, E. Ivanov, S. Kalitsyn, V. Ogievetsky and E. Sokatchev, Unconstrained $N=2$ matter, Yang-Mills and supergravity theories in harmonic superspace, Class. and Quant. Grav. 1 (1984) 469.

[27] B. Zupnik, Harmonic superpotentials and symmetries in gauge theories with eight supercharges, Nucl. Phys. B 554 (1999) 365 hep-th/9902038.

[28] R. Brooks and S.J. Gates Jr., Extended supersymmetry and superBF gauge theories, Nucl. Phys. B 432 (1994) 205 hep-th/9407147.

[29] S. Minwalla, Restrictions imposed by superconformal invariance on quantum field theories, Adv. Theor. Math. Phys. 2 (1998) 781 hep-th/9712074.

[30] M.V. Berry, Quantal phase factors accompanying adiabatic changes, Proc. Roy. Soc. Lond. A 392 (1984) 45.

[31] B. Simon, Holonomy, the quantum adiabatic theorem and berry's phase, Phys. Rev. Lett. 51 (1983) 2167.

[32] J. Wess and J. Bagger, Supersymmetry and supergravity, 2nd edition, Princeton, NJ:

Princeton University Press 1992.

[33] T.T. Wu and C.N. Yang, Dirac monopole without strings: monopole harmonics, Nucl. Phys. B 107 (1976) 365 . 